\title{
Norois
}

Environnement, aménagement, société

\section{Intérêts et limites des statistiques agricoles pour l'étude des structures spatiales produites par l'agriculture en Basse-Normandie}

Interests and limits of agricultural statistics for studying spatial structures produced by agriculture in Normandy

Maxime Marie, Michaël Bermond, Patrick Le Gouée et Daniel Delahaye

\section{OpenEdition}

Journals

Édition électronique

URL : https://journals.openedition.org/norois/1954

DOI : 10.4000 /norois. 1954

ISBN : 978-2-7535-1555-0

ISSN : $1760-8546$

Éditeur

Presses universitaires de Rennes

Édition imprimée

Date de publication : 1 juin 2008

Pagination : 21-33

ISBN : 978-2-7535-0717-3

ISSN : 0029-182X

Référence électronique

Maxime Marie, Michaël Bermond, Patrick Le Gouée et Daniel Delahaye, « Intérêts et limites des statistiques agricoles pour l'étude des structures spatiales produites par l'agriculture en BasseNormandie », Norois [En ligne], 207 | 2008/2, mis en ligne le 01 juin 2010, consulté le 14 janvier 2022. URL : http://journals.openedition.org/norois/1954 ; DOI : https://doi.org/10.4000/norois.1954 


\title{
INTÉRÊTS ET LIMITES DES STATISTIQUES AGRICOLES \\ POUR L'ÉTUDE DES STRUCTURES SPATIALES PRODUITES \\ PAR L'AGRICULTURE EN BASSE-NORMANDIE
}

\author{
Maxime Marie $^{1}$, Michael Bermond ${ }^{2}$, Patrick Le Gouée ${ }^{1}$, Daniel Delahaye ${ }^{1}$ \\ ${ }^{1}$ GÉOPHEN - LETG UMR 6554 CNRS \\ ${ }^{2}$ CRESO - ESO UMR 6590 CNRS \\ (Université de Caen Basse-Normandie), \\ Esplanade de la Paix - 14032 CAEn cedex \\ maxime.marie@unicaen.fr
}

\section{RÉSUMÉ}

Les statistiques agricoles permettent d'aborder de façon intéressante la production de structures spatiales par l'agriculture à l'échelle d'une région, la Basse-Normandie. Les recensements agricoles proposent un grand nombre de variables qui offrent la possibilité de traiter à la fois la structuration de l'espace agricole (principales cultures, surfaces toujours en herbe, etc.) mais aussi les différentes configurations technico-économiques dans lesquelles se trouvent les exploitations agricoles (nomenclature des OTEX, cheptels, UTA). Le recours aux traitements statistiques multivariés permet d'effectuer un travail de typologie qui met en évidence d'importantes différenciations entre les espaces agricoles, des continuités, des discontinuités spatiales et des espaces de transition. Ce type de travail implique cependant une réflexion critique à propos du caractère normatif des nomenclatures statistiques utilisées par le recensement agricole. Par ailleurs, si l'on souhaite aborder pleinement la diversité des exploitations agricoles au sein d'une aire géographique restreinte et leur impact sur l'espace et l'environnement, l'enquête de terrain demeure une étape incontournable pour interpréter et compléter les résultats issus des traitements réalisés à partir des statistiques agricoles.

MOTS CLÉS : Agriculture - Élevage - Structures spatiales - Structures paysagères Environnement - Statistiques agricoles - Exploitations agricoles - Système de production agricole-Basse-Normandie.

\section{ABSTRACT}

Interests and limits of agricultural statistics for studying spatial structures produced by agriculture in Normandy

Agricultural statistics could be an interesting way to work about the spatial structures produced by agriculture on regional scale, in this case Normandy. The agricultural census gives a choice of a large panel of data which allow approaching the agricultural land patterns (main crops, permanent grass, etc.) and the economic configurations of the production system (farm type, cattle and worker). Statistic studies, through multivarious methods, allow a typological work which is interesting because it shows important spatial differentiations, continuities, discontinuities and transitions spaces between agricultural areas. This type of work implies obviously a critical reflexion about the normative character 
of the data from the agricultural census. In addition, if the researcher wishes to have a global approach for the farming system diversity and the agricultural impacts on space and environment in a small area, the local survey is an essential step for understanding and completing results from the statistical approach.

KEY WORDS : Agriculture - Breeding - Spatial Structures - Landscape Structures - Environment - Agricultural Statistics - Farm - Agricultural production System - Normandy.

Les activités agricoles produisent des structures spatiales ${ }^{1}$ qui participent de façon déterminante à la construction des paysages et aux variations de la qualité de la ressource environnementale en milieu rural (Chatellier et Vérité, 2003). L'étude attentive des relations entre l'agriculture, l'espace, les paysages et l'environnement souligne les enjeux scientifiques et sociétaux de la recomposition des rôles sociaux et territoriaux des activités agricoles. La compréhension de ces relations peut se faire de plusieurs manières, et à plusieurs échelles spatiales et temporelles. Le recours aux statistiques agricoles est indispensable si l'on souhaite étudier ces relations à l'échelle régionale, mais les données, tout comme les traitements statistiques retenus, doivent, au préalable, être soumis à un regard critique, tant sur le plan de leur pertinence que sur le plan de leur légitimité (Mathieu et Mordefroid, 1975).

La Basse-Normandie constitue le site-laboratoire de ce travail de recherche, cette région est intéressante car elle concentre de nombreuses zones de contact paysager (bocage/openfield) et une grande variété de systèmes de production agricole (fig. 1). Les espaces d'élevage seront ici étudiés avec une attention particulière ${ }^{2}$. Le croisement des données et le recours à différentes approches méthodologiques constituent un élément central de la méthode développée pour comprendre la production d'espace et de paysage par l'agriculture.

L'objet de cet article est d'aborder l'organisation des structures agricoles ${ }^{3}$ bas-normandes en 2000 à partir de la nomenclature des OTEX (Orientation technico-économique des exploitations) fournies par le recensement agricole, et de s'interroger ainsi sur les intérêts et les limites de ce type de statistiques agricoles. Le croisement de cette nomenclature avec les données relatives aux usages agricoles du sol permettra de construire une typologie synthétique des espaces agricoles bas-normands. Cette dernière ne saurait cependant s'affranchir d'un travail de terrain afin de mieux comprendre les modalités de production et donc de différenciation des espaces agricoles.

Lorsque l'on s'interroge sur l'impact des activités agricoles sur la structuration de l'espace, les paysages et l'environnement à l'échelle régionale, l'utilisation des données relatives à la surface agricole utile (SAU) dans le recensement agricole semble incontournable ${ }^{4}$. Ces données fournissent, en effet, des informations importantes sur les cultures (surfaces cultivées en blé, orge, colza, betteraves, etc.) et sur la conduite des surfaces fourragères (superficie occupée par le maïs fourrage, la prairie temporaire, la STH, etc.). De nombreuses cartes peuvent être produites à partir de ces données puisqu'elles sont mobilisables à différents échelons cartographiques, dont le plus fin est celui de la commune. De nombreux travaux conduits par des géographes s'intéressant aux espaces ruraux et agricoles adoptent ce type d'approche afin de mettre en place des atlas ou

1. Ce terme est ici entendu comme l'ensemble des inscriptions spatiales du fonctionnement des exploitations agricoles (maille parcellaire, combinaisons d'utilisation du sol, réseau bocager, bâti agricole).

2. Recherches effectuées dans le cadre d'une thèse de géographie en cours de réalisation.

3. Ce terme est ici à comprendre comme l'ensemble des caractéristiques économiques, foncières et sociales des systèmes de production agricole.

4. La SAU communale est calculée en additionnant les surfaces des exploitations dont le siège administratif se situe dans la commune en question. Ainsi, la SAU d'une commune peut parfois être supérieure à sa superficie totale, puisque les exploitations exploitent souvent des terres hors du territoire communal. 


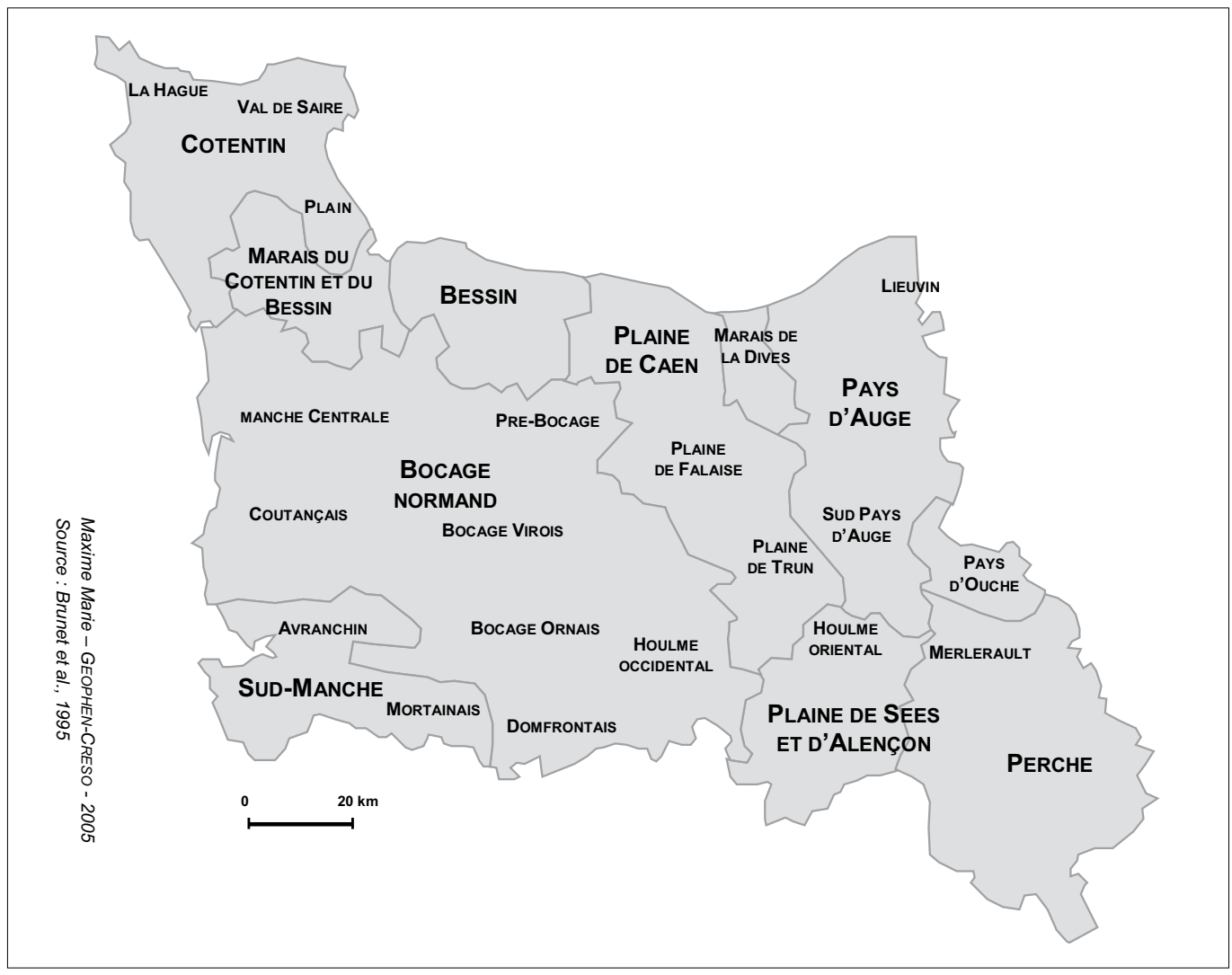

Figure 1 : Les régions agricoles de Basse-Normandie The agricultural areas of «Basse-Normandie»

des inventaires régionaux consistant en une suite de cartes régionales uni-variées (Brunet et al., 1995). Ce type d'approche est évidemment essentiel pour qui s'intéresse aux structures spatiales et paysagères produites par les activités agricoles, mais il demeure insuffisant car une telle problématique nécessite de s'interroger sur les configurations des systèmes d'exploitation agricole qui produisent les espaces agricoles.

Les premières recherches sur les thématiques agricoles ayant des visées typologiques sont maintenant anciennes mais toujours d'actualité sur le plan méthodologique. Les travaux pionniers d'Y. Guermond $(1975,1978)$, par exemple, sont très riches en enseignements car la réflexion sur la nature des variables, leur pertinence et leur représentativité, y est particulièrement aboutie.

\section{Les espaces agricoles bas-normands}

\section{LES NOMENCLATURES OTEX}

Les recensements agricoles fournissent également de nombreuses statistiques sur les structures agricoles (cheptels, équipements, orientations technico-économiques, surface des exploitations, etc.). La nomenclature dite des OTEX permet de classer les exploitations agricoles en fonction des combinaisons de productions animales et/ou végétales. Cette nomenclature intègre les caractéristiques structurelles des exploitations agricoles (surfaces et cheptels) et repose sur la valeur 
monétaire des différents produits de l'exploitation, exprimée à partir de leur contribution à la formation de la marge brute standard $\left(\mathrm{MBS}^{5}\right)$. Le nombre de catégories est important puisqu'il s'élève à 77 types correspondant à 77 combinaisons productives différentes (par exemple pour qu'une exploitation agricole soit classée en OTEX 1310, les céréales, les oléo-protéagineux et les jachères aidées doivent représenter plus des 2/3 de la MBS de l'exploitation).

La principale limite de cette nomenclature réside dans le caractère normatif du classement, établi sur la contribution de chaque production à la formation de la marge brute standard globale de l'exploitation agricole. Aucun critère distinctif de dimension économique ou spatiale n'est en effet intégré à ce mode de catégorisation (Bermond, 2006). Ainsi, une même OTEX, regroupe des exploitations produisant les mêmes types de denrées mais dont l'organisation des systèmes de production peut être radicalement différente. Par exemple, une exploitation laitière intensive de 150 ha pour un quota annuel de 600000 litres de lait sera classée en OTEX 4110, au même titre qu'une exploitation laitière herbagère de 50 ha pour 150000 litres de lait annuel.

Pour caractériser la configuration des structures agricoles bas-normandes en 2000, nous avons créé une nomenclature des combinaisons productives en 14 catégories $^{6}$. Le profil de répartition des OTEX de chaque commune bas-normande a ensuite été traité suivant 3 modalités : le poids démographique des OTEX (pourcentage des exploitations de la commune), le poids économique des OTEX (pourcentage de la MBS communale) et le poids spatial des OTEX (pourcentage de la SAU communale). Dans les trois tableaux de pourcentages, chaque ligne représente une commune et chaque colonne, une catégorie d'OTEX (soit trois tableaux de 1806 lignes et 14 colonnes). Ces trois tableaux ont ensuite été assemblés pour créer un tableau de 5418 lignes et 14 colonnes où chaque commune est représentée trois fois, une fois pour chaque modalité (poids démographique, poids économique et poids spatial). Cette opération permet de réaliser une classification ascendante hiérarchique (CAH) sur l'ensemble des 5418 individus statistiques et d'obtenir une typologie des communes d'après le profil de répartition des exploitations en OTEX. Cette méthode statistique présente l'avantage de mettre en évidence des types communs aux trois modalités de représentation du poids des OTEX dans chaque commune. La comparaison de la catégorisation de chaque commune suivant chacune des modalités est donc directement opérationnelle (Paulus, 2004 ; et Dussol et al., 2004).

\section{UNE GÉOGRAPHIE CONTRASTÉE DES STRUCTURES AGRICOLES}

La classification automatique des communes selon le profil de répartition des OTEX aboutit à une typologie en 11 types (fig. 2-3 - planche II-III, 4 et 5). Trois grands groupes se dégagent nettement : les communes spécialisées vers des orientations productives liées aux céréales et aux grandes cultures, les communes où les systèmes mixtes de polycultures-élevage sont dominants, et les communes où les systèmes d'élevage sont majoritaires. La construction d'une carte pour chaque mode de représentation du profil de répartition des OTEX (poids démographique, poids économique et poids spatial) offre la possibilité d'aborder de façon très différente la configuration des structures agricoles bas-normandes (fig. 2 - planche II).

La figure $2 \mathrm{~A}$ met en évidence une géographie des structures agricoles bas-normandes organisée autour des systèmes spécialisés dans la production de viande bovine dans le Pays d'Auge et le département de la Manche (types C5 et C6), des systèmes de grandes cultures dans la partie centrale de la Plaine de Caen (types A1), des systèmes de polycultures-élevage dans le Perche et la Plaine d'Alençon (type B3). Les profils de répartition des orientations technico-économiques sont ici calculés en pourcentage des exploitations agricoles. La forte représentation des orientations

5. La notion de marge brute standard est assez proche de celle de valeur ajoutée. La MBS est une estimation du potentiel productif d'une exploitation agricole, exprimée en hectare équivalent-blé, c'est-à-dire en nombre d'hectare dégageant la même marge brute.

6. Ce regroupement manuel est fonction de la configuration technico-économique de l'agriculture bas-normande. Ainsi, les postes relatifs aux combinaisons d'élevage et de "polycultures-élevage » sont très représentés, alors que ceux relatifs aux combinaisons de cultures fruitières et maraîchères le sont par exemple nettement moins. 
«viande bovine », dans le Bocage normand notamment, s'explique par le fait qu'un grand nombre de très petites exploitations conduites par des agriculteurs retraités ou pluriactifs fonctionne de manière plus ou moins dynamique avec souvent moins de cinq vaches allaitantes. Si ces systèmes concentrent une forte part des exploitations agricoles, on remarque qu'il n'en va pas de même lorsque l'on s'intéresse à leur poids dans la formation de la MBS.

Type "grandes cultures"

Type A1

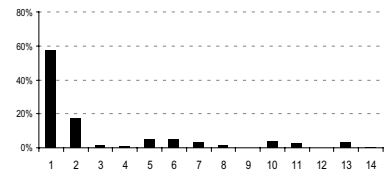

Type A2

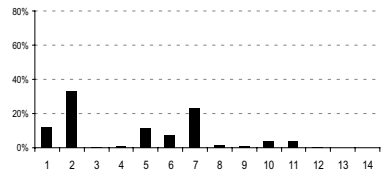

Type "polyculture-élevage"

Type B1

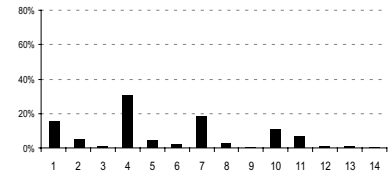

Type B2
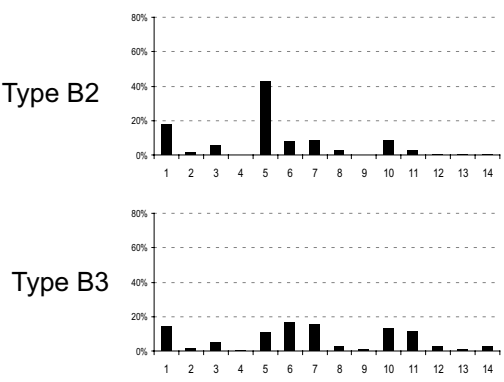

\section{Orientations}

technico-économiques :

1 :céréales
2 :grandes cultures
3 :légumes
4 :polyculture
5 :polyculture bovins-lait
6 :polyculture bovins-viande
7 : bovins-lait

Type "élevage"

Type C1

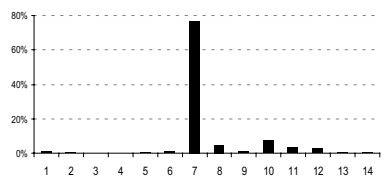

Type C2

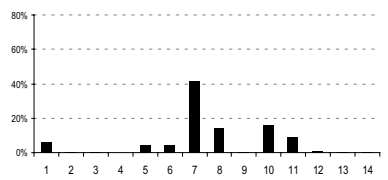

Type C3

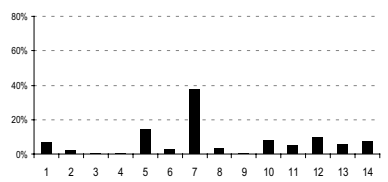

Type C4

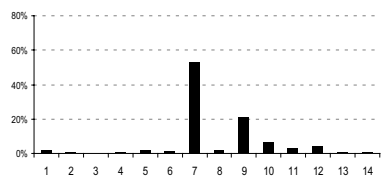

Type C5

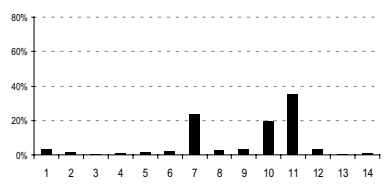

Type C6

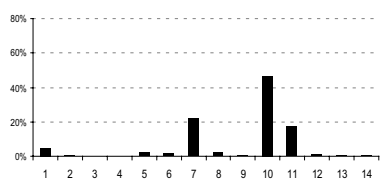

Figure 4 : Typologie des communes bas-normandes suivant le profil de répartition des OTEX en 2000 (légende détaillée) 


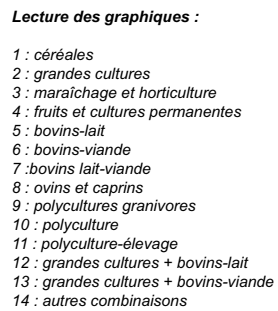

Pôle A : Céréales et grandes cultures
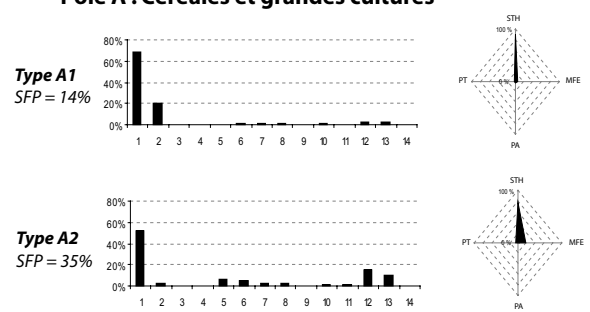

Pôle B : Cultures légumières et maraîchage
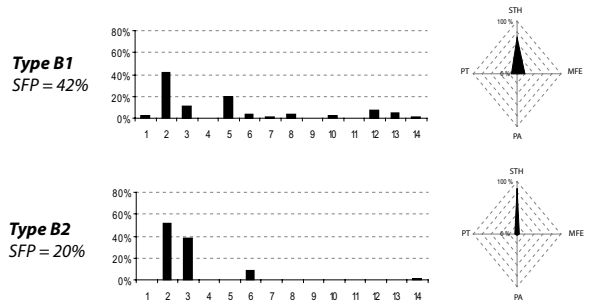

Pôle C : polyculture-élevage

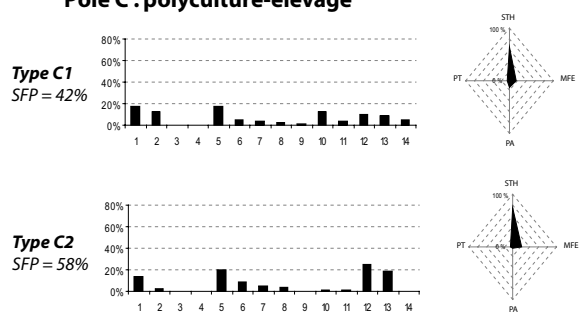

Pôle D : Elevage
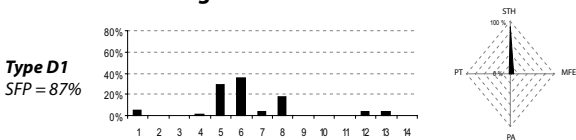

Type D2

$S F P=89 \%$
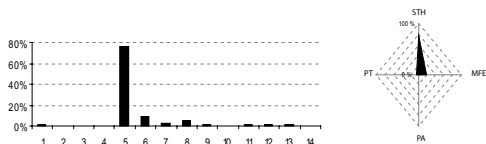

Type D3
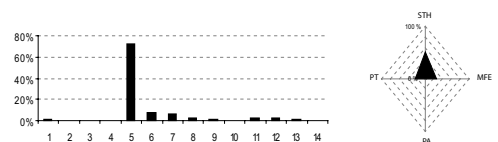

Type D4

$S F P=71 \%$
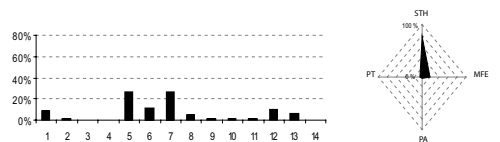

Type D5

$S F P=75 \%$
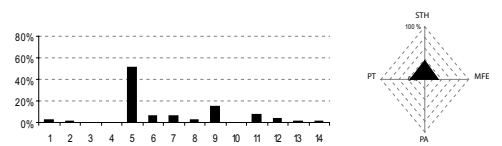

Figure 5 : Typologie des communes bas-normandes suivant le profil technico-économique et l'occupation du sol en 2000 (légende détaillée) use in 2000

Typology of the communes of Normandy based on the OTEX repartition profil and agricultural land

La figure $2 \mathrm{~B}$ présente, en effet, une géographie des productions agricoles très différente de celle de la carte 1. En effet, ce sont ici les systèmes laitiers qui structurent l'espace agricole bas-normand. Les systèmes spécialisés dans la production laitière (types $\mathrm{C} 1$ et $\mathrm{C} 2$ ) sont très bien représentés dans le Pays d’Auge, le Bocage ornais et les deux tiers nord du département de la Manche, car ils concentrent une forte part de la MBS des communes de ces zones (fig. 4).

Les systèmes basés sur les céréales et les grandes cultures étendent leur emprise sur toute la Plaine de Caen et les systèmes légumiers (systèmes à haute valeur ajoutée) sont très présents, notamment sur le littoral du Cotentin (type B1). Enfin, les systèmes associant production laitière et élevage hors-sol (type C4) concernent un grand nombre de communes situées dans le sud du département de la Manche. Ces systèmes concentrent effectivement une forte part de la MBS communale de cette zone, mais les exploitations qui possèdent cette combinaison de production sont souvent de petite dimension foncière; leur poids relatif dans la SAU de ces communes est donc relativement faible. 
La figure $2 \mathrm{C}$ met en lumière une organisation des structures agricoles plus en phase avec notre problématique. En effet, c'est ici la part de la SAU concentrée par chaque poste productif qui participe au classement des communes. Cette approche des systèmes agricoles suivant leur poids spatial permet de brosser le portrait d'une géographie des structures agricoles pertinente mais qui ne peut se passer des analyses conduites suivant les profils de répartition en fonction du poids démographique et économique des différentes OTEX. On remarque ainsi que les systèmes de cultures céréalières et industrielles (type A1 et A2) débordent maintenant la Plaine de Caen jusqu’à en repousser les limites. Les systèmes laitiers spécialisés dominent très nettement dans tout le Bocage normand, et deux pôles de production de viande bovine apparaissent dans le Nord du Pays d'Auge et le Houlme oriental.

La géographie agricole régionale apparaît donc de manière radicalement différente suivant le profil de répartition des OTEX en pourcentage du nombre d'exploitations agricoles, en pourcentage de la MBS communale, ou en pourcentage de la SAU communale. La dernière approche semble être la plus pertinente pour comprendre la contribution des différentes orientations technico-économiques à la structuration des espaces agricoles. Cependant, le caractère normatif de la nomenclature OTEX (pas de prise en compte des dimensions économiques et spatiales des exploitations, ni du mode de conduite du système de production) empêche de se satisfaire d'une telle approche (Cochet et Devienne, 2004). Un croisement des variables relatives aux OTEX et des variables relatives au mode de conduite des surfaces agricoles apparaît comme un passage essentiel pour une caractérisation pertinente des relations entre agriculture et espace à l'échelle régionale.

\section{Vers une approche statistique globale des relations agriculture/espace}

\section{OBJECTIFS ET MÉTHODE DE L'APPROCHE TYPOLOGIQUE}

Pour aborder de la façon la plus complète possible la production de structures spatiales et paysagères par les activités agricoles, la mise en place d'une approche statistique globale de l'agriculture normande est nécessaire. Le choix des variables statistiques et la manière de les traiter sont essentiels pour la pertinence des résultats. Nous avons ainsi choisi d'effectuer une classification ascendante hiérarchique sur le profil communal de répartition des OTEX en pourcentage de la SAU et sur dix variables descriptives des usages agricoles du sol. Ces dix variables se divisent en deux groupes, d'une part celles qui sont relatives à la structuration de la SAU, et celles, plus spécifiques aux espaces d'élevage, qui sont relatives aux modes de conduite de la surface fourragère principale (SFP).

Les six variables descriptives pour la SAU sont : la part de la surface fourragère principale (SFP), la part des surfaces toujours en herbe (STH), la part des céréales, la part des cultures industrielles, la part des légumes frais et la part des cultures permanentes. Les quatre variables relatives au mode de conduite de la SFP sont : la part de la STH, la part du maïs fourrage et ensilage (MFE), la part de la prairie temporaire (PT) et la part de la prairie artificielle (PA).

La classification des communes bas-normandes a donc été réalisée à l'aide d'une CAH selon le profil de répartition des OTEX suivant leur poids spatial et les modes de conduite de la SAU et de la SFP. Une seconde classification suivant la méthode dite des nuées dynamiques a ensuite été appliquée à la partition en onze classes issue de la CAH afin de diminuer la variance intraclasse et donc d'homogénéiser chacune des classes (Bermond, 2004). La classification aboutit à une typologie en onze types structurés en quatre grands ensembles : un pôle « céréales et grandes cultures ", un pôle «cultures légumières et maraîchage », un pôle " polycultures-élevage » et un pôle « élevage» (fig. 4 et 5). 
Maxime Marie, Michael Bermond, Patrick Le Gouée, Daniel Delahaye

\section{LA DIVERSITÉ RÉGIONALE DES SYSTÈMES AGRICOLES}

La géographie des systèmes agricoles qui se dégage de cette typologie est particulièrement intéressante car, au-delà des clivages classiques entre les zones de cultures et les zones d'élevage, apparaissent des espaces de transition, des continuités et des discontinuités géographiques. Par ailleurs, l'intégration des données relatives à la conduite de la SAU et de la SFP permet de différencier plusieurs types d'espaces d'élevage notamment en fonction du degré d'intensification des productions fourragères (fig. 4 et 5). Ce dernier élément permet de palier une des limites majeures de la nomenclature OTEX, celle du défaut de différenciation des systèmes de production sur la base de leur degré d'intensification fourragère.

Les espaces agricoles spécialisés dans les céréales et les grandes cultures se divisent en deux types A1 et A2. Le type A1 regroupe les communes dans lesquelles la SFP représente une part extrêmement faible de la SAU (moins de $15 \%$ ) et où l'association des postes productifs 1 et 2 (céréales et grandes cultures) occupe plus de $85 \%$ de la SAU. Les communes appartenant à ce type se retrouvent exclusivement dans les Plaines de Caen et de Falaise. Le type A2 est assez proche du type précédent à la différence que les postes 12 et 13 (exploitations pratiquant l'élevage bovin en complément des cultures de vente) occupent presque $30 \%$ de la SAU, la SFP occupe donc une part un peu plus importante dans la SAU (35\%). Ce type rassemble un nombre important de communes du Perche ornais et des marges occidentales et méridionales de la Plaine de Caen.

Au sein des zones de cultures légumières et de maraîchage, on distingue deux types B1 et B2. Le type Bl ne concerne qu'une seule commune (Créance); son profil était suffisamment singulier pour conserver ce type, d'autant que cette commune apparaît comme le noyau du petit bassin légumier qui l'enserre. Les autres communes de ce pôle appartiennent au type B2, les exploitations légumières se partagent ici l'espace agricole avec des exploitations de polycultures ou d'élevage bovin. Ce type d'organisation agricole de l'espace se retrouve essentiellement dans le Val de Saire et sur le littoral occidental du département de la Manche.

Les espaces agricoles orientés vers une double spécialisation « polycultures-élevage » se divisent en deux catégories (types C1 et C2). Le type C1 rassemble essentiellement des communes du Perche. Celles-ci ne présentent pas de réelle spécialisation productive, l'association des postes céréales, grandes cultures et polyculture concentre un peu plus de $45 \%$ de la SAU. La part de la SFP dans la SAU y est relativement réduite $(42 \%)$ et est surtout le fait des exploitations laitières. Le type C2 est, quant à lui, davantage marqué par l'orientation vers l'élevage puisque la majorité de la surface agricole est consacrée aux fourrages (58\%). On remarque que les exploitations de polycultures-élevage (postes 12 et 13) concentrent presque $45 \%$ de la SAU et que près de $40 \%$ de cette dernière est utilisé par des postes productifs liés à l'élevage (postes 5, 6, 7 et 8). Les communes appartenant à ce type se retrouvent dans les parties centrale et orientale du département de l'Orne, mais aussi sur les contreforts du Bocage Normand, où elles font figure de zone de transition entre les espaces d'élevage du Massif armoricain et les bas plateaux de la Campagne de Caen.

Enfin, les espaces d'élevage se différencient en cinq types essentiellement sur la base du degré d'intensification fourragère et de spécialisations secondaires différentes.

Le type D1 regroupe les communes du Pays d'Auge et du Houlme oriental dans lesquelles les exploitations orientées « bovins-viande » dominent tout juste celles orientées « bovins-lait ». Dans ces espaces, la SFP occupe souvent plus de $85 \%$ de la SAU et le système fourrager peut y être qualifié d'herbager (la STH occupe $90 \%$ de la SFP).

Les types D2 et D3 présentent quasiment le même profil de répartition des orientations technico-économiques dans la SAU, la seule différence majeure réside dans le niveau d'intensification fourragère, plus élevé dans le Bocage virois et ornais que dans les deux tiers nord du département de la Manche. Par ailleurs, un autre facteur participe à la différenciation de ces deux types : la part de céréales cultivée dans la SAU est plus importante pour les communes appartenant au type D3. 
Le type D4 rassemble un grand nombre de communes du département de l'Orne mais aussi des marges du Massif armoricain. Ce type se caractérise par une part, plus faible que pour les types précédents, de la SAU consacrée à la SFP (71 \%). Ceci s'explique par la présence non négligeable d'exploitations orientées "Céréales et grandes cultures » de grande dimension foncière et associant une production secondaire orientée vers l'élevage (un peu plus de $25 \%$ de la SAU pour l'association des postes 1,2, 12 et 13). Par ailleurs, les communes de ce type font figure d'espaces mixtes car les exploitations spécialisées dans l'élevage montrent une orientation marquée vers les postes «bovins-lait », « bovins-viande » et surtout « lait-viande » (poste 7).

Enfin, le type D5, très caractéristique des communes du Sud Manche, associe une orientation « bovins-lait » avec la présence de nombreux ateliers hors-sol (porcins ou avicoles, poste 9). L'intensification fourragère y est aussi très marquée puisque la SFP est partagée en trois tiers presque égaux entre le maïs-fourrage, la prairie temporaire et la STH.

Cette typologie globale des systèmes de production agricole en Basse-Normandie, s'inscrit bien dans la problématique des relations entre l'agriculture et l'espace. Elle permet de mettre en lumière des différenciations importantes, au sein des espaces d'élevage, qui restent invisibles lorsque l'on étudie séparément les structures agricoles (OTEX) et les usages agricoles du sol. Cependant, ce type de travail, pour être pertinent, ne saurait s'affranchir d'un travail de terrain reposant sur l'enquête auprès des exploitants agricoles, afin de comprendre plus finement les mécanismes de production de structures spatiales par l'agriculture. En effet, l'approche statistique s'appuyant sur les variables du recensement agricole ne permet pas d'aborder (ou seulement de façon très partielle) la diversité des exploitations agricoles présentes dans un espace donné et qui participent au fonctionnement du système agricole local (Dobromez et Bousset, 1996; et Landais, 1998). Dans le Bocage virois par exemple, où une très grande majorité des communes appartiennent au type D3, la part relativement importante des céréales dans la SAU est-elle le fait d'une faible part des exploitations ou de l'ensemble de ces dernières?

Un transfert d'échelle doit donc être réalisé afin d'aborder de façon plus précise l'organisation des exploitations agricoles et les configurations internes de ces dernières. Trois zones majoritairement laitières ont été retenues pour effectuer une enquête de terrain par questionnaire : le Sud Manche (type D5), le Bocage virois (type D3) et le Sud Pays d'Auge (types D2). Dans ces trois zones, les exploitations laitières occupent une part très importante de la SAU, mais les spécialisations secondaires et les modalités de conduite des surfaces fourragères y sont très différentes (fig. 5). Par ailleurs, ces trois espaces connaissent des pressions environnementales d'origine agricole tout à fait variées. Le Sud Manche par exemple est classé, dans le cadre de la directive Nitrates, en «zone en excédents structurels d'azote». Le Bocage Virois est, quant à lui, classé en «zone vulnérable » alors que le Sud du Pays d'Auge ne fait l'objet d'aucune classification particulière (DIREN de Basse-Normandie).

\section{Le transfert d'échelle et l'enquête de terrain : le cas des systèmes laitiers}

\section{L'ENQUÊTE DE TERRAIN}

Le travail de terrain a été réalisé durant l'hiver 2007 dans le Bocage Virois, le Sud Manche et le Sud Pays d'Auge, soit sept ans après la date du recensement agricole de 2000. Cet écart peut paraître important mais plusieurs raisons viennent justifier la pertinence de cette enquête. Tout d'abord, le recensement de 2000 est le dernier en date, et les grandes orientations productives n'ont visiblement pas été bouleversées; c'est surtout la dimension spatiale et économique des exploitations qui a fortement augmenté (c'est pourquoi les variables de taille des structures n'ont pas été intégrées dans les traitements statistiques). De plus, cette enquête offre la possibilité de réactualiser les données de 2000 et de les analyser avec un oeil nouveau.

La distribution des questionnaires s'est faite de manière directe avec les agriculteurs par distribution dans les sièges d'exploitation sans rendez-vous préalablement fixé. Après une courte discussion 
informelle, durant laquelle les objectifs de l'enquête leur ont été expliqués, les questionnaires ont été distribués, accompagnés d'une enveloppe timbrée. 225 questionnaires ont ainsi été délivrés, soit 75 par zone. Le taux de retour est élevé puisqu'il atteint $65 \%, 147$ retours dont 138 complètement exploitables $(61,3 \%)$. Le questionnaire comporte pourtant un nombre élevé de questions et le temps nécessaire pour le remplir est important (entre 25 et 45 minutes). Les exploitants à titre professionnel ${ }^{7}$ (Laurent et Rémy, 2000) ont été particulièrement recherchés car, selon les statistiques, ce sont eux qui concentrent la plus grande partie de la SAU et de la MBS (fig. 3).

Plusieurs parties du questionnaire sont consacrées aux structures spatiales de l'exploitation (usages des surfaces agricoles, évolution de ces dernières depuis l'installation de l'exploitant, statut juridique des terres, mécanismes de mise en œuvre des pratiques agricoles) mais aussi aux orientations productives (productions dominantes, cheptels, équipements, mécanismes de diversification). Les questionnaires ont ensuite été codés et traités statistiquement.

\section{DES SYSTÈMES D’EXPLOITATION CONTRASTÉS}

La compréhension de la production des structures spatiales et paysagères par les exploitations agricoles nécessite de s’interroger sur les éléments qui tendent à les différencier (Bonnamour, 1975). Dans des exploitations où la production laitière est majoritairement dominante (quelques exploitations reposent sur des systèmes «vaches allaitantes », surtout dans le Pays d'Auge), ces critères de différenciation concernent les modes de conduite des surfaces fourragères (niveau d'intensification) et la part des cultures dites « de vente » dans la SAU des exploitations (essentiellement les céréales et le colza, qui peuvent être également autoconsommées dans le système de production). Après avoir transformé les données absolues en pourcentages, les individus statistiques ont été projetés sur un plan composé de deux axes suivant leur niveau d'intensification fourragère et de « céréalisation » (fig. 6).

L'axe des abscisses représente les valeurs « centrées-réduites » de la part occupée par la prairie dans la surface fourragère totale de l'exploitation agricole (indice d'intensification du système fourrager). L'axe des ordonnées, quant à lui, représente les valeurs « centrées-réduites » de la part des céréales dans la surface totale d'exploitation (indice de «céréalisation » du système d'exploitation). Enfin, le diamètre des cercles représentant chaque individu statistique est proportionnel à sa dimension spatiale.

Plusieurs types d'exploitations se dégagent, et l'on distingue évidemment bien les exploitations des trois zones d'étude. Celles du Sud Pays d'Auge sont de dimension spatiale importante, globalement peu intensives et peu «céréalisées ». Les exploitations du Bocage virois sont de dimensions moyennes à grandes, relativement intensives et consacrent des parts plutôt importantes de leur surface aux cultures céréalières. Enfin, les exploitations du Sud Manche sont de tailles globalement assez modestes mais intensives et relativement peu "céréalisées ». Cette réalité globale semble tout à fait en accord avec les éléments de la typologie issue du traitement des données du recensement agricole de 2000. Les résultats de cette enquête mettent aussi en lumière l'extrême diversité des systèmes d'exploitation en présence dans chacune des zones.

Dans le Pays d'Auge méridional, les exploitations utilisant un système fourrager strictement basé sur la prairie permanente sont souvent des systèmes traditionnels de dimension spatiale assez faible, reposant sur la production de viande bovine ${ }^{8}$ et de pommes à cidre issues des prairies complantées de pommiers hautes tiges (cas 1, fig. 6). On trouve aussi dans cette zone, des exploitations laitières de beaucoup plus grande dimension spatiale et économique (quota laitier souvent supérieur à 450000 litres de lait), peu intensives et peu « céréalisées ». Ces systèmes

7. La légitimité de la définition de l'exploitation agricole dite "professionnelle », au sens du recensement agricole, est soumise à de nombreux débats, entre les économistes et les sociologues de l'INRA notamment.

8. Ces systèmes « viande bovine » possèdent deux variantes : système naisseur-engraisseur d'un côté (taurillons vendus pour être abattus), et système allaitant-naisseur de l'autre (veaux, génisses et broutard vendus pour l'engraissement). 
d'exploitation accordent une grande part à la STH dans la SFP, engraissent les taurillons issus du cheptel laitier et vendent les pommes des vergers qui subsistent lorsque ceux-ci sont suffisamment nombreux (cas 2, fig. 6).

Dans le Bocage virois, on remarque facilement un groupe d'exploitations relativement grandes et intensives dans lesquelles la part des cultures céréalières dans la SAU est très importante. Ces exploitations sont toutes des exploitations laitières de grande dimension économique (plus de 500000 litres de quota), et les céréales représentent la spécialisation secondaire du système de production (cas 3, fig. 6).

Enfin, dans le Sud Manche, on remarque un groupe d'exploitations de dimension spatiale relativement faible mais de dimension économique moyenne reposant sur un système laitier conduit de manière assez intensive du fait de la relative faiblesse des surfaces d'exploitation. Le recours à la culture du maïs-fourrage est important (entre 35 et $45 \%$ de la SFP) et ces exploitations possèdent systématiquement un atelier taurillon intensif ou un petit atelier hors-sol complémentaire ${ }^{9}$ (cas 4, fig. 6).

On remarque, par ailleurs, un petit groupe d'exploitations laitières très intensives, de grande dimension foncière et économique (quota de plus de 500000 litres), qui associent systématiquement à la production de lait un atelier porcin hors-sol de dimension importante et un atelier taurillon (cas 5, fig. 6).

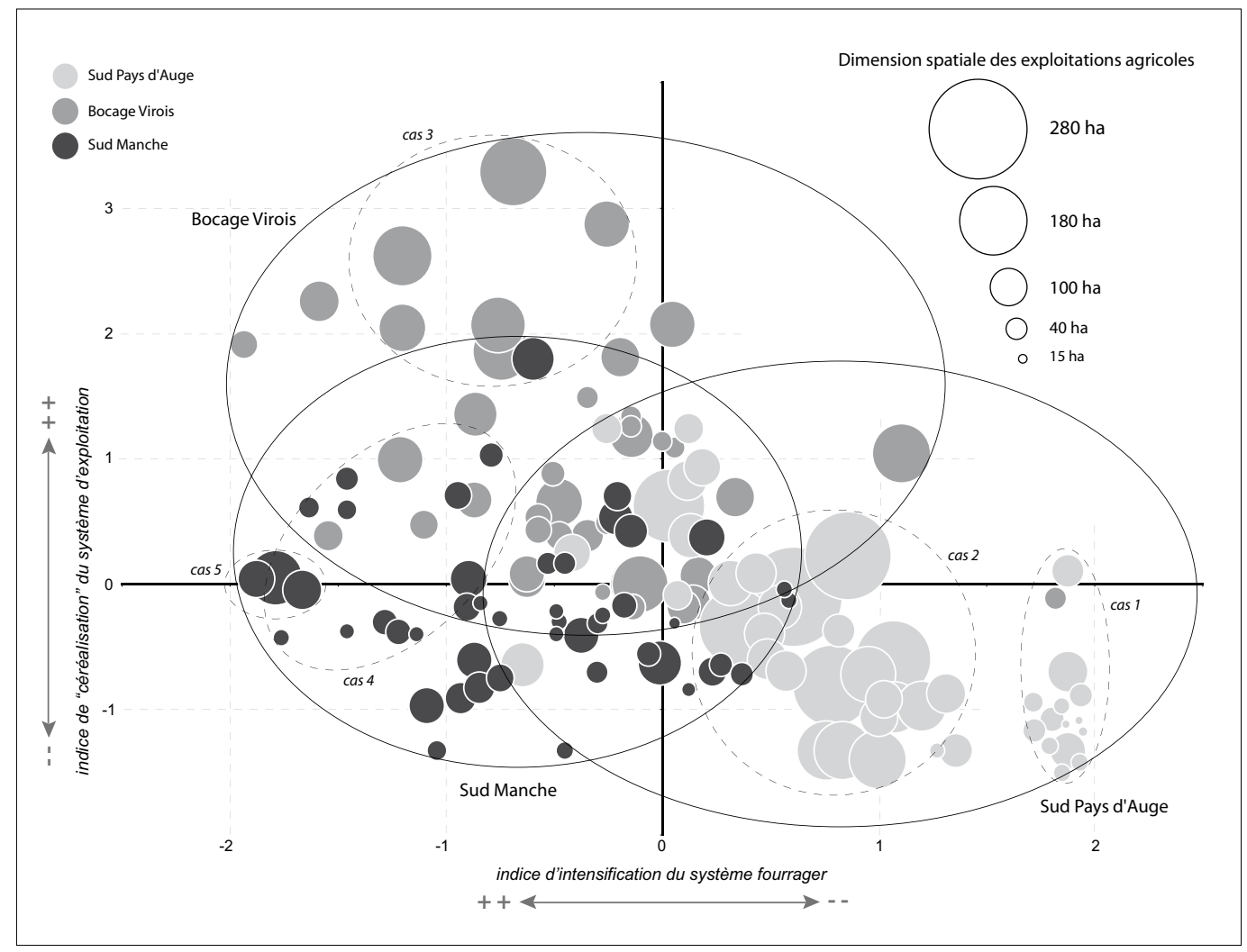

Figure 6 : Les structures spatiales des exploitations agricoles de l'enquête The spatial structures of the farm in the local survey

9. Ce type d'atelier hors-sol est souvent «intégré », c'est-à-dire que les équipements et les intrants sont à la charge d'une entreprise agro-alimentaire, l'agriculteur nourrit les animaux et reçoit une part du prix de vente. 
L'enquête de terrain prouve ici sa complémentarité avec l'étude statistique régionale. La diversité des systèmes d'exploitation, souvent minorée par les approches statistiques classiques, et pourtant à l'origine de l'organisation de l'espace agricole, peut donc être abordée en complément d'une approche plus globale. Le transfert d'échelle prouve ainsi son efficacité dans la compréhension de processus de production des espaces (Thenail, 1996). Les spécificités agricoles, paysagères et environnementales de chacune des zones d'étude s'expliquent ainsi en partie par la présence d'exploitations de grande dimension économique et/ou spatiale ayant un impact fort sur les caractéristiques environnementales et paysagères de l'espace agricole.

\section{Conclusion}

Les statistiques agricoles se révèlent être une source incontournable lorsque l'on souhaite aborder une problématique agricole dans le champ disciplinaire de la géographie. La nomenclature des OTEX est très intéressante car elle permet d'étudier les orientations de production dominante des exploitations. Il faut cependant rester attentif au caractère normatif de cette nomenclature. La dimension foncière, comme la dimension économique et les modes de conduite du système de production (niveau d'intensification) ne sont pas pris en compte par ce type d'indicateur. Par ailleurs, l'approche statistique choisie pour étudier les profils de répartition des OTEX, doit être adaptée à la problématique que ce travail est censé servir. L'étude des OTEX suivant leur poids spatial (la part de chaque poste dans la SAU) apparaît comme l'approche la plus pertinente pour aborder la production de structures spatiales par les activités agricoles. Les autres approches (poids démographique et économique) demeurent néanmoins indispensables pour comprendre et expliquer la structuration des systèmes agricoles.

Le croisement des variables relatives aux orientations technico-économiques et des variables d'utilisation agricole du sol permet la réalisation d'une typologie tout à fait intéressante des espaces agricoles bas-normands. On remarque en effet que de vastes ensembles géographiques se dégagent avec leurs « noyaux durs » et leurs limites (la plaine de Caen, le Pays d'Auge, le Centre Manche). On observe des continuités et des discontinuités spatiales, mais aussi des espaces de transition entre certains grands ensembles (le Pré-Bocage, le Houlme occidentale, le Sud Manche).

Ces éléments permettent d'examiner sous un jour nouveau le découpage des petites régions agricoles en Basse-Normandie. Le traitement des données à l'échelle communale permet de démontrer l'hétérogénéité interne de ces petites régions prétendument homogènes (Klatzmann, 1955). Ce découpage ne peut donc être utilisé comme échelon statistique élémentaire de traitement lorsque l'on souhaite travailler à l'échelle régionale. En revanche, on constate que ce découpage traduit encore assez bien l'hétérogénéité globale des espaces agricoles bas-normands.

Il convient cependant de ne pas oublier qu'il s'agit ici de typologies communales qui ne permettent pas de mettre en lumière la grande diversité des systèmes d'exploitation au sein de chacun des types identifiés à l'échelle régionale. L'enquête par questionnaire révèle en effet la diversité de ces systèmes d'exploitation. Les types exploitations qui participent à la production d'espaces agricoles si contrastés, sont pourtant extrêmement divers. L'influence de certaines catégories d'exploitation, même minoritaires en nombre, peut cependant s'avérer essentielle pour comprendre les mécanismes de production et de différenciation des espaces agricoles.

\section{Bibliographie}

Bermond (M.), 2006. - «1970-2000 : Trente ans d'évolution des systèmes de production agricole dans l'Ouest de la France ", Acteurs et espaces de l'élevage (XVII $-\mathrm{XXI}^{e}$ siècle), bibliothèque d'Histoire Rurale, n 9, PUR, 2006, p. 247-262. 
Bermond (M.), 2004. - Produire et se reproduire dans la crise. Agriculture, Familles, exploitations en Normandie au début du XXI siècle. Essai de géographie sociale. Thèse de doctorat, Université de Caen, 462 p.

Bonnamour (J.) (dir.), 1975. - «Un exemple d'hétérogénéité des systèmes d'exploitation : les exploitations de Domecy-sur-Cure », dans Bonnamour (J.) (dir.), 1975. - Étude géographique des exploitations agricoles : réflexion et propositions méthodologiques, Laboratoire associé de géographie humaine de l'Institut de géographie, Université Paris I-ENS de Fontenay-aux-Roses, p. 27-47.

Brunet (P.), Calmes (R.), Raimbault (M.), 1995. - Atlas de l'agriculture normande, CERVIR-SRSA, PUC, $159 \mathrm{p}$.

Chatellier (V.), Vérité (R.), 2003. - « L'élevage bovin et l'environnement en France : le diagnostic justifiet-il des alternatives techniques », INRA Production Animale, vol. 16, nº 4, p. 231-249.

Cochet (H.), Devienne (S.), 2004 - - « Comprendre l'agriculture d'une région agricole : question de méthode sur l'analyse en termes de système de production », communication au colloque SFER : Les systèmes de production agricole : performances, évolutions et perspectives, diffusion électronique, 16 p. [http://www.sfer. asso.fr/download/71/A_CochetDevienne.pdf].

Dobromez (L.), Bousset (J.-F.), 1996, - Rendre compte de la diversité des exploitations agricoles, coll. « Études du Cemagref, série Gestion des territoires », 318 p.

Dussol (A.-M.), Hilal (M.), Kroll (J.-C.), 2004. - « Permanences et mutations des bassins de production agricole en France de 1988 à 2000 », communication au colloque : Les systèmes de production agricole : performances, évolutions et perspectives, SFER, diffusion électronique, 13 p. [http://www.sfer.asso. fr/download/71/F_Dussol_et_al.pdf].

Guermond (Y.), 1975. - « Les types d'exploitations agricoles en Normandie », dans Bonnamour (J.) (dir.), 1975. - Approche géographique des exploitations agricoles : réflexion et propositions méthodologiques, Laboratoire associé de géographie humaine de l'Institut de géographie, Université Paris I-ENS de Fontenay aux Roses, p. 47-67.

Guermond (Y.), 1978. - Le système de différenciation spatiale en agriculture : la France de l'ouest de 1950 à 1975, Thèse de doctorat, Université de Caen, 376 p.

KLATZMAnN (J.), 1955. - La localisation des cultures et des productions animales en France, Thèse de doctorat, INSEE, imprimerie nationale, Paris, $477 \mathrm{p}$.

LANDAIS (E.), 1998. - « Modelling farm diversity. New approaches to typology building in France », Agricultural System, vol. $58, n^{\circ} 4$, p. 505-527.

Mathieu (N.), Mordefroid (J.-P.), 1975. - « Présentation d'une bibliographie sur les typologies d'exploitation », dans Bonnamour (J.) (dir.), 1975. - Approche géographique des exploitations agricoles, Université Paris I-ENS de Fontenay aux Roses, p. 127-158.

Paulus (F.), 2004. - Coévolution dans les systèmes de villes: croissance et spécialisation des aires urbaines françaises de 1950 à 2000, Thèse de doctorat, Université Paris I Panthéon-Sorbonne, 407 p.

LAurent (C.), RÉmy (J.), 2000. - «L'exploitation agricole en perspective », Le Courrier de l'environnement, $\mathrm{n}^{\circ} 41$, diffusion électronique, 17 p. [http://www.inra.fr/dpenv/laurec41.htm].

Thenail (C.), 1996, - Exploitations agricoles et territoire(s) : contribution à la structuration de la mosä̈que paysagère, Thèse de doctorat, Université de Rennes I, 379 p.

Cet article a été reçu le 30 novembre 2007 et définitivement accepté le 20 avril 2008. 
Planche II (Maxime MARIE et al. - Intérêts et limites des statistiques agricoles...)

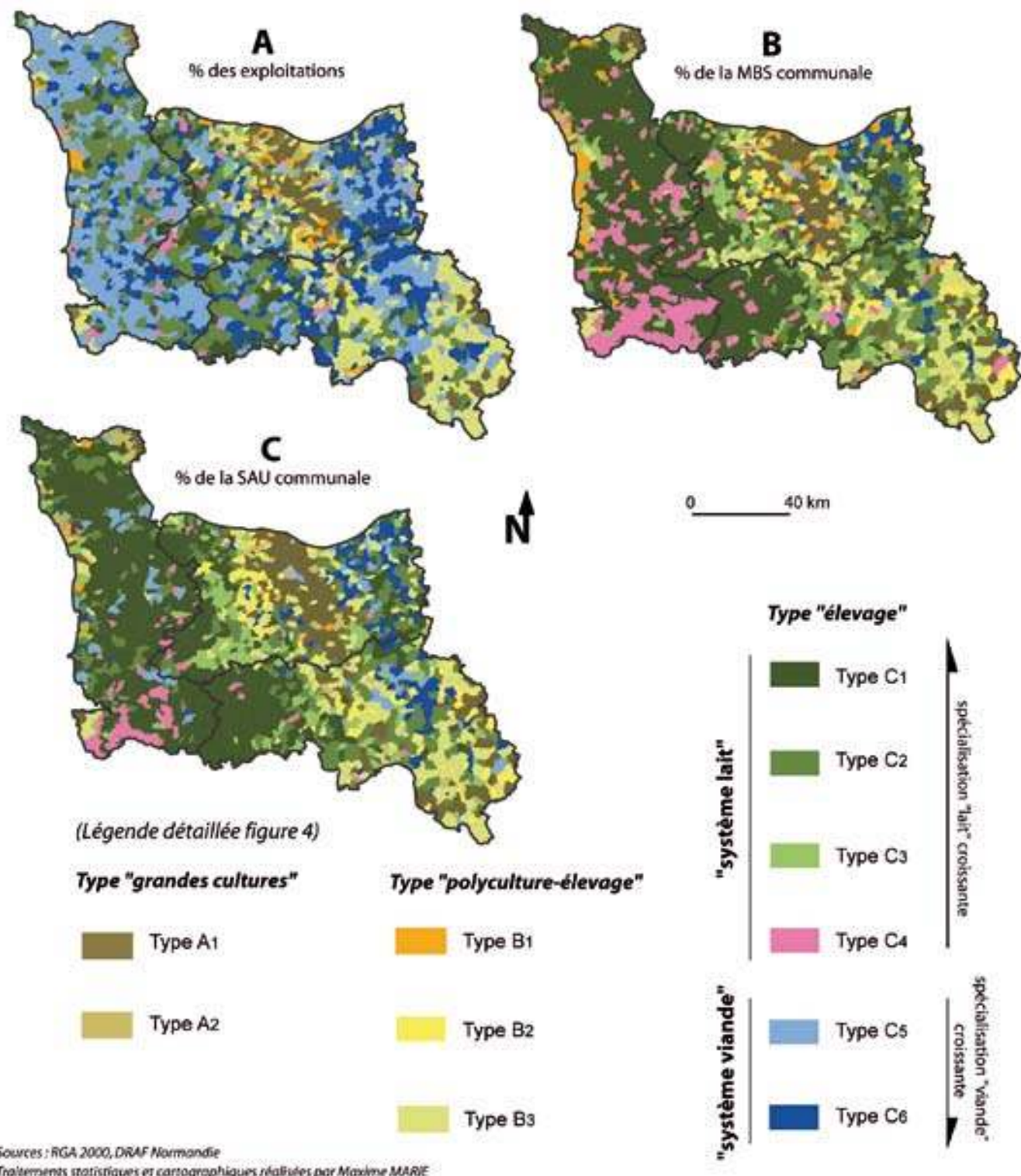

Tratements stotisciques es cartogrephiques realibles par Maxine MAAE

GEOPHEN - CRESO Uhiversite de Com Basse-Namandie, 2007

Figure 2 : Typologie des communes bas-normandes suivant le profil de répartition des OTEX en 2000 Typology of the communes of Normandy based on the OTEX repartition profil in 2000 
Planche III (Maxime MARIE et al. - Intérêts et limites des statistiques agricoles...)

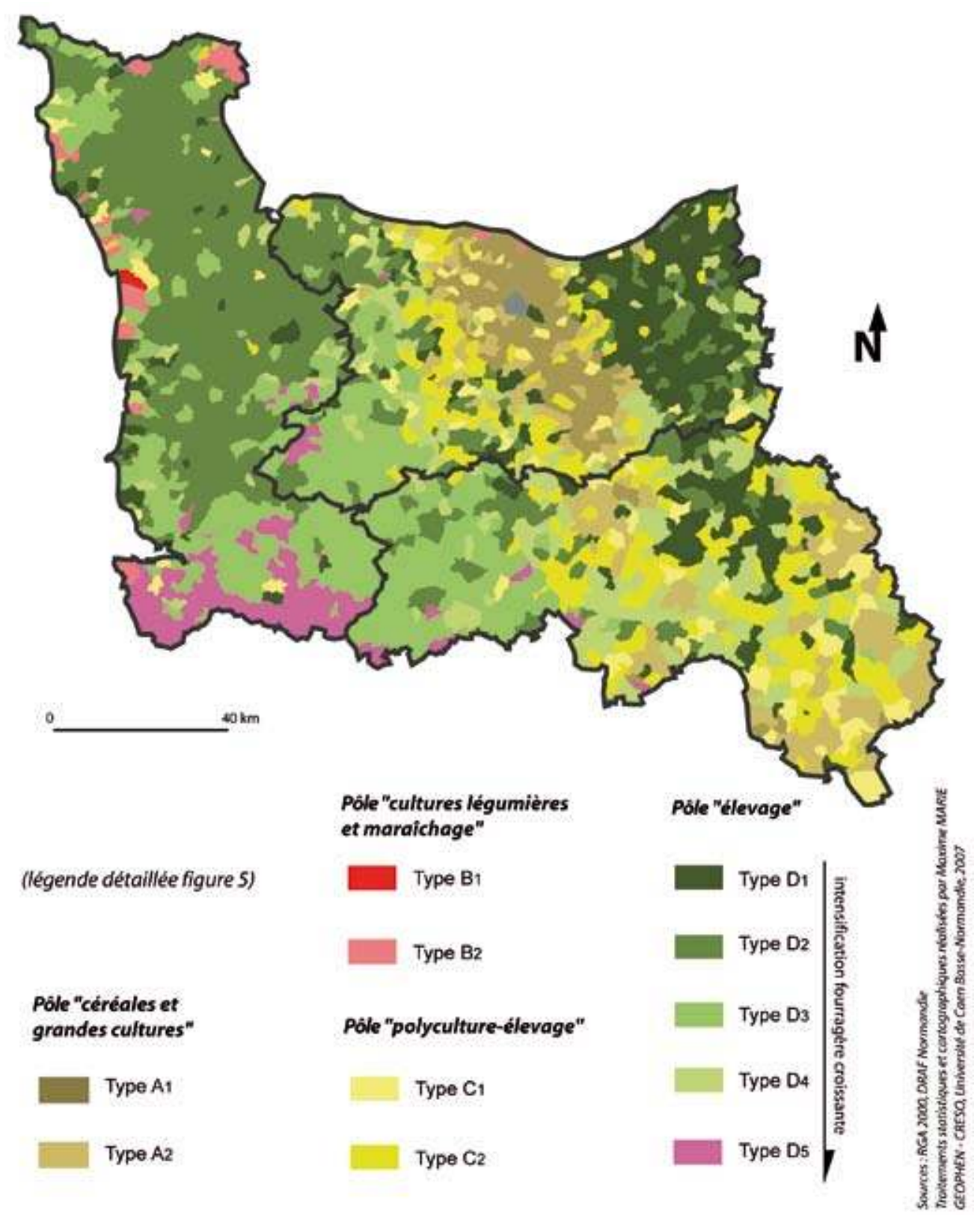

Figure 3 : Typologie des communes bas-normandes suivant le profil technico-économique et l'occupation du sol en 2000 use in 2000

Typology of the communes of Normandy based on the OTEX repartition profil and agricultural land 